\title{
Confidence Intervals for the Scaled Half-Logistic Distribution under Progressive Type-II Censoring
}

Kiran Ganpati Potdar

Department of Statistics, Ajara Mahavidyalaya, Ajara, potdarkiran.stat@gmail.com

D. T. Shirke

Department of Statistics, Shivaji University, Kolhapur, dts_stats@unishivaji.ac.in

Follow this and additional works at: http://digitalcommons.wayne.edu/jmasm

Part of the Applied Statistics Commons, Social and Behavioral Sciences Commons, and the Statistical Theory Commons

\section{Recommended Citation}

Potdar, K. G. \& Shirke, D. T. (2017). Confidence intervals for the scaled half-logistic distribution under progressive Type-II censoring. Journal of Modern Applied Statistical Methods, 16(1), 324-349. doi: 10.22237/jmasm/1493597880

This Regular Article is brought to you for free and open access by the Open Access Journals at DigitalCommons@WayneState. It has been accepted for inclusion in Journal of Modern Applied Statistical Methods by an authorized editor of DigitalCommons@WayneState. 


\section{Confidence Intervals for the Scaled Half-Logistic Distribution under Progressive Type-II Censoring}

\section{Cover Page Footnote}

The first author wishes to thank University Grants Commission, New Delhi, India for providing Fellowship under Faculty Improvement Programme to carry out this research. 


\section{Confidence Intervals for the Scaled Half- Logistic Distribution under Progressive Type-Il Censoring}

\author{
Kiran G. Potdar \\ Ajara Mahavidyalaya \\ Ajara, India
}

\author{
D. T. Shirke \\ Shivaji University \\ Kolhapur, India
}

Confidence interval construction for the scale parameter of the half-logistic distribution is considered using four different methods. The first two are based on the asymptotic distribution of the maximum likelihood estimator (MLE) and log-transformed MLE. The last two are based on pivotal quantity and generalized pivotal quantity, respectively. The MLE for the scale parameter is obtained using the expectation-maximization (EM) algorithm. Performances are compared with the confidence intervals proposed by Balakrishnan and Asgharzadeh via coverage probabilities, length, and coverage-to-length ratio. Simulation results support the efficacy of the proposed approach.

Keywords: Progressively Type-II censoring, EM algorithm, MLE, pivotal quantity, confidence interval, generalized confidence interval, coverage probability, coverage to length ratio, half-logistic distribution

\section{Introduction}

In many life testing situations, an experiment has to be terminated before completion. Because of the various limitations of time and money, testing of life may need to be stopped for some of the units. In day-to-day experiments, incomplete information about the failure times is available, or some of the units must be removed before completion of the experiment. A plan is necessary for removal of the units before the termination of an experiment to save time and cost, which is called the censored data.

Type-I censoring depends on time, where the time is fixed for the termination of experiment. Suppose an observer continues an experiment up to time $T$; lifetimes of units will be known exactly only if these are less than $T$.

Dr. Potdar is an Assistant Professor in the Department of Statistics. Email them at: potdarkiran.stat@gmail.com.Dr. Shirke is a Professor in the Department of Statistics. Email them at:dts_stats@unishivaji.ac.in. 


\section{POTDAR \& SHIRKE}

Failure times of units which have not failed by the time $T$ are not observed. Suppose $n$ units are being tested, but the decision is made to terminate the experiment at time $T$. In this experiment, lifetimes will be known exactly only for those units that fail before time $T$. In Type-I censoring, the number of exact lifetimes observed is random.

A Type-II censoring scheme is often used in life testing experiments where the number of units that can be observed before the termination of the experiment is fixed. In this scheme, only a pre-planned number $m$ out of $n$ units $(m<n)$ are observed. In the case of Type-II censoring, the number of exact lifetimes observed is fixed, but the time required for the termination of the experiment is unknown. In conventional Type-I and Type-II censoring, units are removed from the experiment at the terminal stage, while in a progressive censoring scheme, units are removed at different stages. Progressive censoring schemes can be applied in both Type-I and Type-II censoring schemes. More details about various censoring schemes are available in Lawless (1982).

In an $\left(R_{1}, R_{2}, \ldots, R_{m}\right)$ progressive type-II censoring scheme, the number $m$ and $R_{1}, R_{2}, \ldots, R_{m}$ are fixed before the start of the experiment and $\sum_{i=1}^{m} R_{i}=n-m$. At the first failure, $R_{1}$ units are randomly removed from the remaining $n-1$ units. At the second failure, $R_{2}$ units are randomly removed from the remaining $n-2-R_{1}$ units, etc. At the $m^{\text {th }}$ failure, all the remaining $R_{m}$ units are removed. Here, we observe failure times of $m$ units and the remaining $n-m$ units are removed at different stages of the experiment. In a conventional Type-II censoring scheme, $R_{m}=n-m$ and the rest of the $R_{i}$ are zero.

Consider the problem of interval estimation for the scale parameter of a half-logistic distribution under a progressive Type-II censoring scheme. Progressive Type-II censoring schemes for various lifetime distributions was discussed by Cohen (1963), who introduced progressive Type-II censoring schemes. Mann (1969, 1971), Balakrishnan, Kannan, Lin, and Ng (2003), Balakrishnan, Kannan, Lin, and Wu (2004), Ng (2005), and Ng, Kundu, and Balakrishnan (2006) discussed inference for different lifetime distributions under progressive Type-II censoring schemes. Balakrishnan and Aggarwala (2000) is an excellent reference on progressive censoring. Balakrishnan (2007) studied various distributions and inferential methods for the progressively censored data. Lin and Balakrishnan (2011) discussed the consistency and the asymptotic normality of Maximum Likelihood Estimators (MLEs) based on the progressive Type-II censored samples. Potdar and Shirke $(2013,2014)$ studied inference for the scale parameter of the half logistic and Rayleigh distribution of $k$-unit parallel systems 


\section{I. FOR HALF-LOGISTIC DISTRIBUTION UNDER TYPE-II CENSORING}

based on progressively Type-II censored data. Ghitany, Alqallaf, and Balakrishnan (2014) discussed estimation of the parameters of Gompertz distributions based on progressively Type-II censored samples. Sultan, Alsadat, and Kundu (2014) studied estimation for the inverse Weibull parameters under progressive Type-II censoring.

As far as the half-logistic distribution is concerned, Balakrishnan and Puthenpura (1986) discussed the best linear unbiased estimation of location and scale parameters. Balakrishnan and Wong (1991) computed the approximate Maximum Likelihood Estimator (AMLE) for the location and scale parameters of the half-logistic distribution. Balakrishnan and Chan (1992) studied estimation for the scale parameter of the half-logistic distribution. Kim and Han (2010) used importance sampling methods to obtain a Bayes estimator for the scale parameter of the half-logistic distribution under progressively Type-II censored samples. Jang, Park, and Kim (2011) studied estimation of the scale parameter of the halflogistic distribution with a multiply Type-II censored sample. Rastogi and Tripathi (2014) studied estimation of parameter and reliability for the exponentiated half-logistic distribution.

The likelihood equation of a half-logistic distribution with scale parameter does not have a closed form solution to obtain MLE. In most of the reported work, an AMLE of the scale parameter is obtained. Following this approach, Balakrishnan and Asgharzadeh (2005) and Wang (2009) reported inference for the scale parameter of a half-logistic distribution based on progressive Type-II censored samples.

Balakrishnan and Asgharzadeh (2005) showed that, if the relative sample fraction is small, then the coverage probability of the confidence interval (CI) based on asymptotic normality of the MLE is unsatisfactory. Wang (2009) paid more attention to length of CI and gave a shorter length CI. Dempster, Laird, and Rubin (1977) introduced the expectation-maximization (EM) algorithm to obtain the MLE for the incomplete data. McLachlan and Krishnan (1997) gave more details about the EM algorithm. Here, the MLE is computed using the EM algorithm, and the focus is on both the coverage probability and length of CI.

Assume that $n$ units having half-logistic lifetime distribution are put on test

and failure times of $\sum_{i=1}^{m} R_{i}=n-m$ units are censored. Lifetimes of these censored units are unknown. Consider the censored data as missing data and use the EM algorithm to compute the MLE. As indicated in Potdar and Shirke (2014), the EM algorithm gives improved inferential results. 


\section{Model and Estimation of the Scale Parameter}

Suppose progressively Type-II censored data are obtained from the scaled halflogistic distribution with probability density function

$$
\mathrm{f}(x ; \lambda)=\frac{1}{\lambda} \frac{2 \mathrm{e}^{-x / \lambda}}{\left(1+\mathrm{e}^{-x / \lambda}\right)^{2}}, \quad x \geq 0, \lambda>0
$$

and cumulative distribution function

$$
\mathrm{F}(x ; \lambda)=\left[\frac{1-\mathrm{e}^{-x / \lambda}}{1+\mathrm{e}^{-x / \lambda}}\right], \quad x \geq 0, \lambda>0
$$

Suppose $n$ units are under test and lifetimes of $m$ units are observed under progressive Type-II censoring. Suppose $\left(R_{1}, R_{2}, \ldots, R_{m}\right)$, a progressive censoring scheme, is used. The observed lifetimes $x_{(1)}, x_{(2)}, \ldots, x_{(m)}$ are the progressively Type-II censored sample. The likelihood function for the observed data is given by (Balakrishnan \& Aggarwala, 2000)

$$
\mathrm{L}(\lambda)=C \prod_{i=1}^{m} \mathrm{f}\left(x_{(i)} ; \lambda\right)\left[1-\mathrm{F}\left(x_{(i)} ; \lambda\right)\right]^{R_{i}}
$$

where

$$
C=n \prod_{j=1}^{m-1}\left(n-j-\sum_{i=1}^{j} R_{i}\right)
$$

\section{Maximum Likelihood Estimation}

Suppose $\mathbf{z}_{1}, \mathbf{z}_{2}, \ldots, \mathbf{z}_{m}$ are the censored data. Note $\mathbf{z}_{i}$ is a vector with $R_{i}$ element corresponding to $R_{i}$ removed units after the $i^{\text {th }}$ failure is observed $(i=1,2, \ldots, m)$. The censored data $\mathbf{Z}=\left(\mathbf{z}_{1}, \mathbf{z}_{2}, \ldots, \mathbf{z}_{m}\right)$ can be considered to be the missing data and $\mathbf{X}=\left(x_{(1)}, x_{(2)}, \ldots, x_{(m)}\right)$ the observed data. $\mathbf{W}=(\mathbf{X}, \mathbf{Z})$ is the complete data set to be used for drawing inference for the scale parameter. The complete log-likelihood function can be written as 


$$
L_{c}=-n \log (\lambda)+\sum_{i=1}^{m} \log \left(\frac{2 \mathrm{e}^{-x_{i} / \lambda}}{\left(1+\mathrm{e}^{-x_{i} / \lambda}\right)^{2}}\right)+\sum_{i=1}^{m} \sum_{j=1}^{R_{i}} \log \left(\frac{2 \mathrm{e}^{-z_{i j} / \lambda}}{\left(1+\mathrm{e}^{-z_{i j} / \lambda}\right)^{2}} \mid z_{i j}>x_{i}\right)
$$

By differentiating $L_{c}$ with respect to $\lambda$,

$$
\frac{d L_{c}}{d \lambda}=-\frac{n}{\lambda}+\frac{1}{\lambda^{2}} \sum_{i=1}^{m} \frac{x_{i}\left(1-\mathrm{e}^{-x_{i} / \lambda}\right)}{1+\mathrm{e}^{-x_{i} / \lambda}}+\frac{1}{\lambda^{2}} \sum_{i=1}^{m} \sum_{j=1}^{R_{i}}\left(\frac{z_{i j}\left(1-\mathrm{e}^{-z_{i j} / \lambda}\right)}{1+\mathrm{e}^{-z_{i j} / \lambda}} \mid z_{i j}>x_{i}\right)
$$

The EM algorithm suggested by Dempster et al. (1977) was used to compute the MLE. For the E step in the EM algorithm, the expectation of $Z_{i j}$ was taken. Hence, the above equation becomes

$$
\frac{d L_{c}}{d \lambda}=-\frac{n}{\lambda}+\frac{1}{\lambda^{2}} \sum_{i=1}^{m} \frac{x_{i}\left(1-\mathrm{e}^{-x_{i} / \lambda}\right)}{1+\mathrm{e}^{-x_{i} / \lambda}}+\frac{1}{\lambda^{2}} \sum_{i=1}^{m} R_{i} \mathrm{a}\left(x_{i}, \lambda\right)
$$

where

$$
\begin{aligned}
\mathrm{a}\left(x_{i}, \lambda\right) & =\mathrm{E}\left(\frac{z_{i j}\left(1-\mathrm{e}^{-z_{i j} / \lambda}\right)}{1+\mathrm{e}^{-z_{i j} / \lambda}} \mid z_{i j}>x_{i}\right)=\int_{x_{i}}^{\infty} \frac{z\left(1-\mathrm{e}^{-z_{i j} / \lambda}\right)}{1+\mathrm{e}^{-z_{i j} / \lambda}} \frac{\mathrm{f}(z)}{1-\mathrm{F}\left(x_{i}\right)} d z \\
& =\frac{1+\mathrm{e}^{-x_{i} / \lambda}}{\lambda \mathrm{e}^{-x_{i} / \lambda}} \int_{x_{i}}^{\infty} \frac{z \mathrm{e}^{-z / \lambda}\left(1-\mathrm{e}^{-z / \lambda}\right)}{\left(1+\mathrm{e}^{-z / \lambda}\right)^{3}} d z
\end{aligned}
$$

Solving equation (4) is the M step.

The Newton-Raphson method was used to solve equation (4) by taking the least square estimate as an initial value. $\mathrm{Ng}$ (2005) discussed estimation of model parameters of modified Weibull distributions based on progressively Type-II censored data, where the empirical distribution function is computed as

$$
\hat{\mathrm{F}}\left(x_{(i)}\right)=1-\prod_{j=1}^{i}\left(1-\hat{p}_{j}\right)
$$




\section{POTDAR \& SHIRKE}

with

$$
\hat{p}_{j}=\frac{1}{n-p_{j}^{*}}, p_{j}^{*}=\sum_{k=2}^{j} R_{k-1}-j+1, \quad j=1,2, \ldots, m
$$

The estimate of the parameters can be obtained by the least squares fit of simple linear regression

$$
y_{i}=\beta x_{(i)}
$$

with $\beta=-1 / \lambda$,

$$
\begin{aligned}
& y_{i}=\ln \left[\frac{1-\frac{\hat{\mathrm{F}}\left(x_{(i-1)}\right)+\hat{\mathrm{F}}\left(x_{(i)}\right)}{2}}{1+\frac{\hat{\mathrm{F}}\left(x_{(i-1)}\right)+\hat{\mathrm{F}}\left(x_{(i)}\right)}{2}}\right], \quad i=1,2, \ldots, m \\
& \hat{\mathrm{F}}\left(x_{(0)}\right)=0
\end{aligned}
$$

The least square estimate of $\lambda$ is given by

$$
\hat{\lambda}_{0}=-\frac{\sum_{i=1}^{m} x_{(i)}^{2}}{\sum_{i=1}^{m} x_{(i)} y_{i}}
$$

While obtaining the MLE $\hat{\lambda}_{n}$ of the scale parameter $\lambda$, the above approach was adopted, where $\hat{\lambda}_{0}$ was taken as an initial value of $\lambda$ in the Newton-Raphson method. It will be shown that the MLE $\hat{\lambda}_{n}$ exits and is unique. From equation (2),

$$
\mathrm{L}(\lambda)=C \prod_{i=1}^{m} \frac{2^{R_{i}+1}}{\lambda} \frac{\mathrm{e}^{-\left(R_{i}+1\right) x_{i} / \lambda}}{\left(1-\mathrm{e}^{-x_{i} / \lambda}\right)^{R_{i}+2}}
$$

where $C$ is defined as above. 


$$
\frac{d \log \mathrm{L}}{d \lambda}=-\frac{n}{\lambda}+\frac{1}{\lambda^{2}} \sum_{i=1}^{m}\left(R_{i}+1\right) x_{i}-\frac{1}{\lambda^{2}} \sum_{i=1}^{m} \frac{\left(R_{i}+2\right) x_{i} \mathrm{e}^{-x_{i} / \lambda}}{1+x_{i} \mathrm{e}^{-x_{i} / \lambda}}=0
$$

Define

$$
\mathrm{g}(\lambda)=\frac{-\lambda^{2} d \log \mathrm{L}}{d \lambda}=\frac{d \log \mathrm{L}}{d \lambda}=n \lambda-\sum_{i=1}^{m}\left(R_{i}+1\right) x_{i}+\sum_{i=1}^{m} \frac{\left(R_{i}+2\right) x_{i} \mathrm{e}^{-x_{i} / \lambda}}{1+x_{i} \mathrm{e}^{-x_{i} / \lambda}}=0
$$

Note

$$
\lim _{\lambda \rightarrow 0} g(\lambda)<0, \lim _{\lambda \rightarrow \infty} g(\lambda)>0, \text { and } \mathrm{g}^{\prime}(\lambda)>0
$$

Therefore, the MLE, a solution to $\mathrm{g}(\lambda)=0$, exists and is unique.

\section{Fisher Information}

We compute observed Fisher information using the idea of the missing information principle of Louis (1982). Thus, observed information $=$ complete information - missing information. Write this as

$$
\mathrm{I}_{x}(\lambda)=\mathrm{I}_{w}(\lambda)-\mathrm{I}_{w \mid x}(\lambda)
$$

In the following, we obtain complete and missing information given by

$$
\mathrm{I}_{w}(\lambda)=-\mathrm{E}\left[\frac{d^{2} \mathrm{~L}}{d \lambda^{2}}\right]
$$

where, $\mathrm{L}$ is the $\log$-likelihood function of the complete data. By differentiating $\mathrm{L}$ with respect to $\lambda$ twice

$$
\frac{d^{2} \mathrm{~L}}{d \lambda^{2}}=\frac{n}{\lambda^{2}}-\frac{2}{\lambda^{4}} \sum_{i=1}^{n} \frac{x_{i}^{2} \mathrm{e}^{-x_{i} / \lambda}}{\left(1+\mathrm{e}^{-x_{i} / \lambda}\right)^{2}}-\frac{2}{\lambda^{3}} \sum_{i=1}^{n} \frac{x_{i}\left(1-\mathrm{e}^{-x_{i} / \lambda}\right)}{1+x_{i} \mathrm{e}^{-x_{i} / \lambda}}
$$

The complete information is given by 


\section{POTDAR \& SHIRKE}

$$
\mathrm{I}_{w}(\lambda)=-\frac{n}{\lambda^{2}}+\frac{2}{\lambda^{4}} \sum_{i=1}^{n} \mathrm{E}\left[\frac{X_{i}^{2} \mathrm{e}^{-X_{i} / \lambda}}{\left(1+\mathrm{e}^{-X_{i} / \lambda}\right)^{2}}\right]+\frac{2}{\lambda^{3}} \sum_{i=1}^{n} E\left[\frac{X_{i}\left(1-\mathrm{e}^{-X_{i} / \lambda}\right)}{1+x_{i} \mathrm{e}^{-X_{i} / \lambda}}\right]
$$

Missing information is given by

$$
\mathrm{I}_{W \mid X}(\lambda)=\sum_{i=1}^{m} R_{i} \mathrm{I}_{W \mid X}^{(i)}(\lambda)=-\sum_{i=1}^{m} \sum_{j=1}^{R_{i}} \mathrm{E}_{Z \mid X}\left[\frac{d^{2} \log \left(\mathrm{f}\left(Z_{i j} \mid X_{i}, \lambda\right)\right)}{d \lambda^{2}}\right]
$$

\section{Consider}

$$
\mathrm{f}_{z \mid X}\left(Z_{i j} \mid X_{i}, \lambda\right)=\frac{\mathrm{f}\left(z_{i j} ; \lambda\right)}{1-\mathrm{F}\left(x_{i} ; \lambda\right)}=\frac{\frac{1}{\lambda} \frac{2 \mathrm{e}^{-z_{i j} / \lambda}}{\left(1+\mathrm{e}^{-z_{i j} / \lambda}\right)^{2}}}{1-\left[\frac{1-\mathrm{e}^{-x_{i} / \lambda}}{1+\mathrm{e}^{-x_{i} / \lambda}}\right]}
$$

Therefore,

$$
\begin{gathered}
\log \mathrm{f}=-\log \lambda+\log \left[\frac{2 e^{-z_{i j} / \lambda}}{\left(1+e^{-z_{i j} / \lambda}\right)^{2}}\right]-\log \left[1-\left(\frac{1-e^{-x_{i} / \lambda}}{1+e^{-x_{i} / \lambda}}\right)\right] \\
\frac{d \log \mathrm{f}}{d \lambda}=-\frac{1}{\lambda}+\frac{z_{i j}\left(1-\mathrm{e}^{-z_{i j} / \lambda}\right)}{\lambda^{2}\left(1+\mathrm{e}^{-z_{i j} / \lambda}\right)}-\frac{x_{i}}{\lambda^{2}\left(1+\mathrm{e}^{-x_{i} / \lambda}\right)}
\end{gathered}
$$

and

$$
\frac{d^{2} \log \mathrm{f}}{d \lambda^{2}}=\frac{1}{\lambda^{2}}-\frac{2 z_{i j}^{2} \mathrm{e}^{-z_{i j} / \lambda}}{\lambda^{4}\left(1+\mathrm{e}^{-z_{i j} / \lambda}\right)^{2}}-\frac{2 z_{i j}\left(1-\mathrm{e}^{-z_{i j} / \lambda}\right)}{\lambda^{3}\left(1+\mathrm{e}^{-z_{i j} / \lambda}\right)}+\frac{2 x_{i}^{2} \mathrm{e}^{-x_{i} / \lambda}}{\lambda^{4}\left(1+\mathrm{e}^{-x_{i} / \lambda}\right)^{2}}+\frac{2 x_{i}}{\lambda^{3}\left(1+\mathrm{e}^{-x_{i} / \lambda}\right)}
$$

\section{Hence}




$$
\begin{aligned}
\mathrm{I}_{W \mid X}(\lambda)= & \sum_{i=1}^{m} R_{i} \mathrm{I}_{W \mid X}^{(i)}(\lambda) \\
= & -\frac{n-m}{\lambda^{2}}+\frac{2}{\lambda^{4}} \sum_{i=1}^{m} \sum_{j=1}^{R_{i}} \mathrm{E}\left[\frac{z_{i j}^{2} \mathrm{e}^{-z_{i j} / \lambda}}{\left(1+\mathrm{e}^{-z_{i j} / \lambda}\right)^{2}}\right]+\frac{2}{\lambda^{3}} \sum_{i=1}^{m} \sum_{j=1}^{R_{i}} \mathrm{E}\left[\frac{z_{i j}\left(1-\mathrm{e}^{-z_{i j} / \lambda}\right)}{1+\mathrm{e}^{-z_{i j} / \lambda}}\right] \\
& -\frac{1}{\lambda^{4}} \sum_{i=1}^{m} \frac{R_{i} x_{i}^{2} \mathrm{e}^{-x_{i} / \lambda}}{\left(1+\mathrm{e}^{-x_{i} / \lambda}\right)^{2}}-\frac{2}{\lambda^{3}} \sum_{i=1}^{m} \frac{R_{i} x_{i}}{\left(1+\mathrm{e}^{-x_{i} / \lambda}\right)}
\end{aligned}
$$

\section{Confidence Intervals Based on MLE and log-Transformed MLE}

\section{Confidence Interval Based on MLE}

Let $\hat{\lambda}_{n}$ be the MLE of $\lambda$ and

$$
\hat{\sigma}^{2}\left(\hat{\lambda}_{n}\right)=\frac{1}{\mathrm{I}\left(\hat{\lambda}_{n}\right)}
$$

be the estimated asymptotic variance of $\hat{\lambda}_{n}$. Therefore, a $100(1-\alpha) \%$ asymptotic CI for $\lambda$ based on asymptotic normality of $\hat{\lambda}_{n}$ is given by

$$
\left(\hat{\lambda}_{n}-\tau_{\alpha / 2} \sqrt{\hat{\sigma}^{2}\left(\hat{\lambda}_{n}\right)}, \hat{\lambda}_{n}+\tau_{\alpha / 2} \sqrt{\hat{\sigma}^{2}\left(\hat{\lambda}_{n}\right)}\right)
$$

where $\tau_{\alpha / 2}$ is the upper $100(\alpha / 2)^{\text {th }}$ percentile of the standard normal distribution.

\section{Confidence Interval Based on log-Transformed MLE}

Meeker and Escobar (1998) reported the asymptotic CI for $\lambda$ based on $\log \left(\hat{\lambda}_{n}\right)$. An approximate $100(1-\alpha) \% \mathrm{CI}$ for $\log (\lambda)$ is

$$
\left(\log \left(\hat{\lambda}_{n}\right)-\tau_{\alpha / 2} \sqrt{\hat{\sigma}^{2}\left(\log \left(\hat{\lambda}_{n}\right)\right)}, \log \left(\hat{\lambda}_{n}\right)+\tau_{\alpha / 2} \sqrt{\hat{\sigma}^{2}\left(\log \left(\hat{\lambda}_{n}\right)\right)}\right)
$$




\section{POTDAR \& SHIRKE}

where $\hat{\sigma}^{2}\left(\log \left(\hat{\lambda}_{n}\right)\right)$ is the estimated asymptotic variance of $\log \left(\hat{\lambda}_{n}\right)$, which is approximated by

$$
\hat{\sigma}^{2}\left(\log \left(\hat{\lambda}_{n}\right)\right) \approx \frac{\hat{\sigma}^{2}\left(\hat{\lambda}_{n}\right)}{\hat{\lambda}_{n}^{2}}
$$

Hence, an approximate $100(1-\alpha) \% \mathrm{CI}$ for $\lambda$ is

$$
\left(\hat{\lambda}_{n} \mathrm{e}^{\left(-\frac{\tau_{\alpha / 2} \sqrt{\hat{\sigma}^{2}\left(\hat{\lambda}_{n}\right)}}{\hat{\lambda}_{n}}\right)}, \hat{\lambda}_{n} \mathrm{e}^{\left(\frac{\tau_{\alpha / 2} \sqrt{\hat{\sigma}^{2}\left(\hat{\lambda}_{n}\right)}}{\hat{\lambda}_{n}}\right)}\right)
$$

\section{Confidence Interval Based on Pivotal and Generalized Pivotal Quantity}

Consider two exact CIs based on the pivotal quantities. To define these CIs, show that the distribution of $V=\hat{\lambda} / \lambda$ is free from $\lambda$, where $\hat{\lambda}$ is the MLE of $\lambda$, based on the complete data. In the following lemma, it is proved that $V$ is a pivot, following Gulati and Mi (2006):

Lemma 1: The distribution of $V$ is free from $\lambda$.

Proof: Consider the probability density function of the half-logistic distribution with scale parameter $\lambda$ :

$$
\mathrm{f}(x, \lambda)=\frac{2 \mathrm{e}^{-x / \lambda}}{\lambda\left(1+e^{-x / \lambda}\right)^{2}}, \quad x \geq 0, \lambda>0
$$

Then the log-likelihood function becomes

$$
\mathrm{L}=-n \log (\lambda)+n \log (2)-\frac{1}{\lambda} \sum_{i=1}^{n} x_{i}-2 \sum_{i=1}^{n} \log \left(1+\mathrm{e}^{-x_{i} / \lambda}\right)
$$

$d \mathrm{~L} / d \lambda=0$ gives the following equation: 


$$
\sum_{i=1}^{n} x_{i}-2 \sum_{i=1}^{n} \frac{x_{i} \mathrm{e}^{-x_{i} / \lambda}}{1+\mathrm{e}^{-x_{i} / \lambda}}=n \lambda
$$

The solution of the above equation is the MLE of $\lambda$ ( say $\hat{\lambda}$ ). Hence

$$
\begin{gathered}
\sum_{i=1}^{n} x_{i}-2 \sum_{i=1}^{n} \frac{x_{i} \mathrm{e}^{-x_{i} / \hat{\lambda}}}{1+\mathrm{e}^{-x_{i} / \hat{\lambda}}}=n \hat{\lambda} \\
\sum_{i=1}^{n} \frac{x_{i}}{\lambda}-2 \sum_{i=1}^{n} \frac{x_{i}}{\lambda} \frac{\mathrm{e}^{\frac{-x_{i} \lambda}{\lambda \hat{\lambda}}}}{1+\mathrm{e}^{\frac{-x_{i} \lambda}{\lambda \hat{\lambda}}}}=\frac{n \hat{\lambda}}{\lambda}
\end{gathered}
$$

Let $\xi=\lambda / \hat{\lambda}$ and $Y_{i}=X_{i} / \lambda$. Then

$$
\begin{aligned}
\sum_{i=1}^{n} y_{i}-2 \sum_{i=1}^{n} y_{i} \frac{\mathrm{e}^{-y_{i} \xi}}{1+\mathrm{e}^{-y_{i} \xi}} & =n \xi^{-1} \\
\frac{1}{n} \sum_{i=1}^{n} y_{i}-\frac{2}{n} \sum_{i=1}^{n} y_{i} \frac{\mathrm{e}^{-y_{i} \xi}}{1+\mathrm{e}^{-y_{i} \xi}}-\xi^{-1} & =0
\end{aligned}
$$

Note that $Y_{1}, Y_{2}, \ldots, Y_{n}$ is a random sample from the half-logistic distribution with parameter $\lambda=1$. Therefore, the distribution of $\xi=\lambda / \hat{\lambda}$ is independent of $\lambda$. Hence the proof.

Lemma 2: $\quad$ The distribution of $V$ under progressive Type-II censored data from the half-logistic distribution with scale parameter $\lambda$ is free from $\lambda$.

Proof: $\quad$ This is similar to Lemma 1 and hence is omitted.

This property of the MLE will be used to derive the confidence interval based on pivot and generalized pivot quantity methods.

Remark: $\quad V$ is also a pivot for $k$-unit parallel and $k$-unit series systems.

\section{Confidence Interval Based on Pivotal Quantity}

From Lemma 2, the distribution of $V$ is free from $\lambda$. Define $a$ and $b$ such that 


\section{POTDAR \& SHIRKE}

$$
\mathrm{P}(a<V<b)=1-\alpha
$$

Therefore we obtain the following as a CI for $\lambda$ :

$$
\left(\frac{\hat{\lambda}}{b}, \frac{\hat{\lambda}}{a}\right)
$$

The constants $a$ and $b$ are obtained using Monte Carlo simulation by using the following algorithm:

\section{Algorithm to Obtain Percentiles of V}

1. Input $\alpha, N, m$, and progressive Type-II censoring scheme $\left(R_{1}, R_{2}, \ldots, R_{m}\right)$.

2. Generate a progressive Type-II censored random sample of size $m$ using censoring scheme $\left(R_{1}, R_{2}, \ldots, R_{m}\right)$ from the half-logistic distribution with parameter $\lambda=1$.

3. Obtain a MLE of $\lambda$ ( say $\hat{\lambda}$ ) using the EM algorithm.

4. Repeat steps 2 and $3 N$ times so as to get $\hat{\lambda}_{1}, \hat{\lambda}_{2}, \ldots, \hat{\lambda}_{N}$.

5. Arrange the $\hat{\lambda}_{i}$ in an increasing order. Denote them by $\hat{\lambda}_{(1)}, \hat{\lambda}_{(2)}, \ldots, \hat{\lambda}_{(N)}$.

6. Compute $a=\hat{\lambda}_{([(\alpha / 2) N])}$ and $b=\hat{\lambda}_{([(1-\alpha / 2) N])}$.

\section{Confidence Interval Based on Generalized Pivotal Quantity}

The concept of a generalized confidence interval (GCI) is introduced by Weerahandi (1993). Let $x$ denote the observed value of $X$. To construct a GCI for $\lambda$, first define a generalized pivotal quantity (GPQ), $\mathrm{T}(X ; x, \lambda)$, which is a function of the random variable $X$, its observed value $x$, and the parameter $\lambda$. A quantity $\mathrm{T}(X ; x, \lambda)$ is required to satisfy the following two conditions:

i) For a fixed $x$, the probability distribution of $\mathrm{T}(X ; x, \lambda)$ is free of unknown parameters.

ii) The observed value of $\mathrm{T}(X ; x, \lambda)$, namely $\mathrm{T}(x ; x, \lambda)$, is simply $\lambda$.

Let $\mathrm{T}_{\alpha}$ be the $100 \alpha^{\text {th }}$ percentile of $\mathrm{T}$. Then $\mathrm{T}_{\alpha}$ becomes the $100(1-\alpha) \%$ lower bound for $\lambda$. Therefore a $100(1-\alpha) \%$ two-sided GCI for parameter $\lambda$ is given by 


$$
\left(\mathrm{T}_{\alpha / 2}, \mathrm{~T}_{1-\alpha / 2}\right)
$$

Define the GPQ as

$$
\mathrm{T}(X ; x, \lambda)=\frac{\hat{\lambda}_{0}}{\hat{\lambda} / \lambda}
$$

where $\hat{\lambda}_{0}$ is the MLE obtained using observed data. Note:

i) The distribution of $\mathrm{T}(X ; x, \lambda)$ is free from $\lambda$, which follows from Lemma 2 , and

ii) $\mathrm{T}(x ; x, \lambda)=\lambda$, since for the observed data, $\hat{\lambda}=\hat{\lambda}_{0}$.

A GCI based on $\mathrm{T}(X ; x, \lambda)$ is obtained by using following algorithm:

\section{Algorithm to Obtain CI for $\lambda$ using GPQ}

1. Input $\alpha, N, m$, and progressive Type-II censoring scheme $\left(R_{1}, R_{2}, \ldots, R_{m}\right)$.

2. Generate a progressive Type-II censored random sample of size $m$ from the half-logistic distribution with an unknown parameter $\lambda$.

3. Based on the data in step 2, obtain a MLE of $\lambda$ (say $\hat{\lambda}_{0}$ ) using the EM algorithm.

4. Generate a progressive Type-II censored random sample of size $m$ from the half-logistic distribution with parameter $\lambda=1$.

5. Obtain a MLE of $\lambda$ ( say $\hat{\lambda}_{i}$ ) using the EM algorithm for step 4 data.

6. Compute $T_{i}=\hat{\lambda}_{0} / \hat{\lambda}_{i}$.

7. Repeat steps 4 to $6 N$ times, so as to get $T_{1}, T_{2}, \ldots, T_{N}$.

8. Arrange the $T_{i}$ in an increasing order. Denote them by $T_{(1)}, T_{(2)}, \ldots, T_{(N)}$.

9. Compute a $100(1-\alpha) \%$ CI for $\lambda$ as $\left(T_{([(\alpha / 2) N]}, T_{([(1-\alpha / 2) N])}\right)$.

\section{Simulation Study}

The CIs given in (10) to (13) will now be compared with the CIs given by Balakrishnan and Asgharzadeh (2005) and Wang (2009). A simulation study was 


\section{POTDAR \& SHIRKE}

carried out to study the performance of each of the CIs. Asymptotic CIs based on MLE, log-transformed MLE, and GPQ are compared through length and confidence level. Balakrishnan and Sandhu (1995) presented an algorithm for sample generation from progressively Type-II censored schemes. This algorithm was used to generate samples from a half-logistic distribution. Consider the 34 different progressively Type-II censored schemes compiled in Table 1.

\section{Algorithm}

1. Generate i.i.d. observations $\left(W_{1}, W_{2}, \ldots, W_{m}\right)$ from $\mathrm{U}(0,1)$.

2. For censoring scheme $\left(R_{1}, R_{2}, \ldots, R_{m}\right)$,

$$
E_{i}=\frac{1}{\left(i+R_{m}+R_{m-1}+\ldots+R_{m-i+1}\right)}
$$

for $i=1,2, \ldots, m$.

3. Set $V_{i}=W_{i}^{E_{i}}$ for $i=1,2, \ldots, m$.

4. Set $U_{i}=1-\left(V_{m} \cdot V_{m-1} \cdot \ldots \cdot V_{m-i+1}\right)$ for $i=1,2, \ldots, m$. Then $\left(U_{1}, U_{2}, \ldots, U_{m}\right)$ is the uniform $(0,1)$ progressively Type-II censored sample.

5. For given values of the parameter $\lambda$, set

$$
x_{(i)}=-\lambda \log \left[\frac{1-U_{i}}{1+U_{i}}\right]
$$

for $i=1,2, \ldots, m$.

Then $\left(x_{(1)}, x_{(2)}, \ldots, x_{(m)}\right)$ is the required progressively Type-II censored sample from the half-logistic distribution. In Table 1, censoring scheme $(a, b, c, d)$ stands for $R_{1}=a, R_{2}=b, R_{3}=c$, and $R_{4}=d$. A similar meaning holds for schemes described through completely specified vector, while scheme $(10,9 * 0)$ means $R_{1}=10$ and remaining nine $R_{i}$ are zero, i.e. $R_{2}=R_{3}=R_{4}=\ldots=R_{10}=0$. A simulation was carried out with $\lambda=1$. For each particular progressive censoring scheme, 5,000 sets of observations are generated. The CIs based on asymptotic normal distributions of the MLE and logtransformed MLE are derived. 


\section{I. FOR HALF-LOGISTIC DISTRIBUTION UNDER TYPE-II CENSORING}

Table 1. Censoring schemes

\begin{tabular}{|c|c|c|c|c|}
\hline Scheme No. & $n$ & $m$ & $m / n$ & Scheme \\
\hline [1] & 10 & 4 & 0.2500 & $(0,0,0,6)$ \\
\hline [2] & 10 & 4 & 0.2500 & $(6,0,0,0)$ \\
\hline [3] & 10 & 5 & 0.5000 & $(0,0,0,0,5)$ \\
\hline [4] & 10 & 5 & 0.5000 & $(5,0,0,0,0)$ \\
\hline [5] & 15 & 4 & 0.2667 & $(0,0,0,11)$ \\
\hline [6] & 15 & 4 & 0.2667 & $(11,0,0,0)$ \\
\hline [7] & 15 & 5 & 0.3333 & $(0,0,0,0,10)$ \\
\hline [8] & 15 & 5 & 0.3333 & $(10,0,0,0,0)$ \\
\hline [9] & 15 & 5 & 0.3333 & $(0,10,0,0,0)$ \\
\hline [10] & 15 & 5 & 0.3333 & $(0,0,10,0,0)$ \\
\hline [11] & 15 & 5 & 0.3333 & $(2,2,2,2,2)$ \\
\hline [12] & 15 & 5 & 0.3333 & $(4,4,2,0,0)$ \\
\hline [13] & 20 & 5 & 0.2500 & $(0,0,0,0,15)$ \\
\hline [14] & 20 & 5 & 0.2500 & $(15,0,0,0,0)$ \\
\hline [15] & 20 & 5 & 0.2500 & $(5,5,5,0,0)$ \\
\hline [16] & 20 & 5 & 0.2500 & $(3,3,3,3,3)$ \\
\hline [17] & 20 & 5 & 0.2500 & $(0,15,0,0,0)$ \\
\hline [18] & 20 & 5 & 0.2500 & $(5,10,0,0,0)$ \\
\hline [19] & 20 & 10 & 0.5000 & $\left(9^{*} 0,10\right)$ \\
\hline [20] & 20 & 10 & 0.5000 & $\left(10,9^{*} 0\right)$ \\
\hline [21] & 25 & 5 & 0.2000 & $(0,0,0,0,20)$ \\
\hline [22] & 25 & 5 & 0.2000 & $(20,0,0,0,0)$ \\
\hline [23] & 25 & 10 & 0.4000 & $\left(9^{\star} 0,15\right)$ \\
\hline [24] & 25 & 10 & 0.4000 & $\left(15,9^{*} 0\right)$ \\
\hline [25] & 25 & 15 & 0.6000 & $\left(14^{*} 0,10\right)$ \\
\hline [26] & 25 & 15 & 0.6000 & $\left(10,14^{\star} 0\right)$ \\
\hline [27] & 50 & 20 & 0.4000 & $\left(19^{*} 0,30\right)$ \\
\hline [28] & 50 & 20 & 0.4000 & $\left(30,19^{*} 0\right)$ \\
\hline [29] & 50 & 25 & 0.5000 & $\left(24^{*} 0,25\right)$ \\
\hline [30] & 50 & 25 & 0.5000 & $\left(25,24^{\star} 0\right)$ \\
\hline [31] & 100 & 20 & 0.2000 & $\left(19^{\star} 0,80\right)$ \\
\hline [32] & 100 & 20 & 0.2000 & $\left(80,19^{*} 0\right)$ \\
\hline [33] & 100 & 50 & 0.5000 & $\left(49^{*} 0,50\right)$ \\
\hline [34] & 100 & 50 & 0.5000 & $\left(50,49^{*} 0\right)$ \\
\hline
\end{tabular}


POTDAR \& SHIRKE

Table 2. Simulated coverage probabilities for confidence intervals

\begin{tabular}{|c|c|c|c|c|c|c|c|c|c|c|}
\hline \multirow[b]{2}{*}{ Scheme } & \multicolumn{2}{|c|}{$\mathbf{C}_{1}$} & \multicolumn{2}{|c|}{$\mathrm{C}_{3}$} & \multicolumn{2}{|c|}{$\mathrm{C}_{4}$} & \multicolumn{2}{|c|}{$\mathrm{C}_{5}$} & \multicolumn{2}{|c|}{$\mathrm{C}_{6}$} \\
\hline & $90 \%$ & $95 \%$ & $90 \%$ & $95 \%$ & $90 \%$ & $95 \%$ & $90 \%$ & $95 \%$ & $90 \%$ & $95 \%$ \\
\hline$[1]$ & 0.8100 & 0.8396 & 0.8108 & 0.8470 & 0.8710 & 0.9176 & 0.8944 & 0.9458 & 0.8992 & 0.9474 \\
\hline [2] & 0.8300 & 0.8640 & 0.8338 & 0.8676 & 0.8804 & 0.9282 & 0.9072 & 0.9514 & 0.8986 & 0.9464 \\
\hline [3] & 0.8288 & 0.8638 & 0.8330 & 0.8684 & 0.8768 & 0.9256 & 0.8968 & 0.9462 & 0.9025 & 0.9503 \\
\hline [4] & 0.8290 & 0.8688 & 0.8382 & 0.8768 & 0.8814 & 0.9286 & 0.9014 & 0.9528 & 0.9036 & 0.9494 \\
\hline [5] & 0.8204 & 0.8508 & 0.8160 & 0.8500 & 0.8786 & 0.9204 & 0.8978 & 0.9476 & 0.9016 & 0.9518 \\
\hline [6] & 0.8350 & 0.8650 & 0.8364 & 0.8706 & 0.8830 & 0.9306 & 0.8978 & 0.9528 & 0.8948 & 0.9468 \\
\hline [7] & 0.8194 & 0.8582 & 0.8278 & 0.8640 & 0.8736 & 0.9230 & 0.8998 & 0.9522 & 0.9058 & 0.9548 \\
\hline [8] & 0.8360 & 0.8686 & 0.8418 & 0.8778 & 0.8834 & 0.9284 & 0.9006 & 0.9528 & 0.8998 & 0.9482 \\
\hline [9] & 0.8370 & 0.8684 & 0.8398 & 0.8724 & 0.8794 & 0.9240 & 0.9050 & 0.9526 & 0.8986 & 0.9498 \\
\hline [10] & 0.8354 & 0.8656 & 0.8364 & 0.8666 & 0.8780 & 0.9306 & 0.8946 & 0.9456 & 0.8978 & 0.9506 \\
\hline [11] & 0.8262 & 0.8596 & 0.8308 & 0.8684 & 0.8822 & 0.9274 & 0.9022 & 0.9494 & 0.9050 & 0.9518 \\
\hline [12] & 0.8354 & 0.8650 & 0.8408 & 0.8798 & 0.8896 & 0.9336 & 0.9014 & 0.9514 & 0.8934 & 0.9486 \\
\hline [13] & 0.8318 & 0.8626 & 0.8418 & 0.8750 & 0.8842 & 0.9348 & 0.9002 & 0.9504 & 0.8966 & 0.9520 \\
\hline [14] & 0.8474 & 0.8806 & 0.8474 & 0.8834 & 0.8866 & 0.9342 & 0.8960 & 0.9474 & 0.8974 & 0.9462 \\
\hline [15] & 0.8368 & 0.8740 & 0.8388 & 0.8716 & 0.8752 & 0.9250 & 0.8974 & 0.9528 & 0.9008 & 0.9482 \\
\hline [16] & 0.8308 & 0.8632 & 0.8312 & 0.8664 & 0.8816 & 0.9260 & 0.9048 & 0.9532 & 0.8950 & 0.9496 \\
\hline [17] & 0.8432 & 0.8724 & 0.8492 & 0.8818 & 0.8870 & 0.9296 & 0.9004 & 0.9504 & 0.9000 & 0.9464 \\
\hline [18] & 0.8318 & 0.8690 & 0.8390 & 0.8756 & 0.8788 & 0.9260 & 0.8944 & 0.9488 & 0.8998 & 0.9500 \\
\hline [19] & 0.8592 & 0.8954 & 0.8790 & 0.9122 & 0.8902 & 0.9416 & 0.8960 & 0.9510 & 0.8950 & 0.9458 \\
\hline [20] & 0.8680 & 0.9068 & 0.8706 & 0.9098 & 0.8864 & 0.9358 & 0.9002 & 0.9528 & 0.8958 & 0.9418 \\
\hline [21] & 0.8196 & 0.8544 & 0.8280 & 0.8606 & 0.8764 & 0.9284 & 0.8990 & 0.9496 & 0.8976 & 0.9492 \\
\hline [22] & 0.8372 & 0.8720 & 0.8400 & 0.8712 & 0.8764 & 0.9304 & 0.8972 & 0.9542 & 0.8970 & 0.9504 \\
\hline [23] & 0.8640 & 0.9072 & 0.8636 & 0.8994 & 0.8858 & 0.9364 & 0.8976 & 0.9490 & 0.8980 & 0.9454 \\
\hline [24] & 0.8774 & 0.9128 & 0.8780 & 0.9132 & 0.8964 & 0.9434 & 0.8904 & 0.9466 & 0.9010 & 0.9512 \\
\hline [25] & 0.8714 & 0.9160 & 0.8770 & 0.9158 & 0.8948 & 0.9432 & 0.8926 & 0.9448 & 0.9006 & 0.9466 \\
\hline [26] & 0.8822 & 0.9210 & 0.8848 & 0.9242 & 0.8996 & 0.9504 & 0.9008 & 0.9492 & 0.8938 & 0.9468 \\
\hline [27] & 0.8844 & 0.9246 & 0.8790 & 0.9212 & 0.8914 & 0.9388 & 0.9002 & 0.9502 & 0.8970 & 0.9472 \\
\hline [28] & 0.8852 & 0.9302 & 0.8880 & 0.9292 & 0.8952 & 0.9470 & 0.9084 & 0.9532 & 0.8948 & 0.9496 \\
\hline
\end{tabular}




\section{I. FOR HALF-LOGISTIC DISTRIBUTION UNDER TYPE-II CENSORING}

Table 2, continued.

\begin{tabular}{|c|c|c|c|c|c|c|c|c|c|c|}
\hline \multirow[b]{2}{*}{ Scheme } & \multicolumn{2}{|c|}{$\mathbf{C}_{1}$} & \multicolumn{2}{|c|}{$\mathbf{C}_{3}$} & \multicolumn{2}{|c|}{$\mathbf{C}_{4}$} & \multicolumn{2}{|c|}{$\mathbf{C}_{5}$} & \multicolumn{2}{|c|}{$\mathbf{C}_{6}$} \\
\hline & $90 \%$ & $95 \%$ & $90 \%$ & $95 \%$ & $90 \%$ & $95 \%$ & $90 \%$ & $95 \%$ & $90 \%$ & $95 \%$ \\
\hline [29] & 0.8904 & 0.9276 & 0.8950 & 0.9360 & 0.9022 & 0.9494 & 0.9024 & 0.9466 & 0.8948 & 0.9504 \\
\hline [30] & 0.8896 & 0.9348 & 0.8918 & 0.9374 & 0.8982 & 0.9484 & 0.9044 & 0.9530 & 0.8978 & 0.9478 \\
\hline [31] & 0.8920 & 0.9324 & 0.8856 & 0.9248 & 0.8962 & 0.9460 & 0.9008 & 0.9526 & 0.8968 & 0.9486 \\
\hline [32] & 0.8864 & 0.9306 & 0.8876 & 0.9336 & 0.8972 & 0.9478 & 0.9062 & 0.9534 & 0.8958 & 0.9478 \\
\hline [33] & 0.8930 & 0.9374 & 0.8938 & 0.9408 & 0.8998 & 0.9454 & 0.8958 & 0.9446 & 0.9046 & 0.9530 \\
\hline [34] & 0.8924 & 0.9416 & 0.9010 & 0.9452 & 0.9026 & 0.9522 & 0.8948 & 0.9448 & 0.9070 & 0.9544 \\
\hline
\end{tabular}

Table 3. The expected lengths of confidence intervals

\begin{tabular}{|c|c|c|c|c|c|c|c|c|c|c|c|c|}
\hline \multirow[b]{2}{*}{ Scheme } & \multicolumn{2}{|c|}{$\mathbf{C}_{1}$} & \multicolumn{2}{|c|}{$\mathbf{C}_{2}$} & \multicolumn{2}{|c|}{$\mathrm{C}_{3}$} & \multicolumn{2}{|c|}{$\mathrm{C}_{4}$} & \multicolumn{2}{|c|}{$\mathbf{C}_{5}$} & \multicolumn{2}{|c|}{$\mathbf{C}_{6}$} \\
\hline & $90 \%$ & $95 \%$ & $90 \%$ & $95 \%$ & $90 \%$ & $95 \%$ & $90 \%$ & $95 \%$ & $90 \%$ & $95 \%$ & $90 \%$ & $95 \%$ \\
\hline$[1]$ & 2.0913 & 2.7742 & 2.0330 & 2.7028 & 1.3723 & 1.6352 & 1.4919 & 1.8397 & 2.0003 & 2.6406 & 2.0432 & 2.7096 \\
\hline [2] & 2.0150 & 2.6663 & 1.9223 & 2.5345 & 1.3790 & 1.6432 & 1.4943 & 1.8403 & 1.9281 & 2.5360 & 1.9254 & 2.5328 \\
\hline [3] & 1.6829 & 2.2413 & 1.6495 & 2.1395 & 1.2142 & 1.4468 & 1.2952 & 1.5849 & 1.6353 & 2.1214 & 1.6562 & 2.1440 \\
\hline [4] & 1.6656 & 2.1061 & 1.5932 & 2.0518 & 1.2246 & 1.4592 & 1.3051 & 1.5965 & 1.5883 & 2.0467 & 1.5690 & 2.0143 \\
\hline [5] & 2.1526 & 2.8298 & 2.1217 & 2.8244 & 1.4289 & 1.7026 & 1.5625 & 1.9313 & 2.1204 & 2.8675 & 2.0944 & 2.7809 \\
\hline [6] & 2.0219 & 2.8139 & 1.9415 & 2.5615 & 1.3863 & 1.6519 & 1.5039 & 1.8530 & 1.9146 & 2.5256 & 1.9121 & 2.5117 \\
\hline [7] & 1.8253 & 2.3360 & 1.7234 & 2.2392 & 1.2655 & 1.5079 & 1.3562 & 1.6627 & 1.7120 & 2.2377 & 1.7132 & 2.2203 \\
\hline [8] & 1.7290 & 2.2818 & 1.6054 & 2.0685 & 1.2395 & 1.4770 & 1.3220 & 1.6177 & 1.6076 & 2.0631 & 1.5954 & 2.0493 \\
\hline [9] & 1.6816 & 2.1968 & 1.6431 & 2.1214 & 1.2488 & 1.4880 & 1.3343 & 1.6339 & 1.6136 & 2.0929 & 1.6358 & 2.1071 \\
\hline [10] & 1.8064 & 2.2591 & 1.6754 & 2.1675 & 1.2566 & 1.4973 & 1.3445 & 1.6474 & 1.6653 & 2.1710 & 1.6636 & 2.1482 \\
\hline [11] & 1.7245 & 2.2904 & 1.6782 & 2.1775 & 1.2430 & 1.4812 & 1.3285 & 1.6270 & 1.6886 & 2.2053 & 1.6426 & 2.1253 \\
\hline [12] & 1.6759 & 2.1434 & 1.6449 & 2.1252 & 1.2481 & 1.4872 & 1.3333 & 1.6326 & 1.6374 & 2.1200 & 1.6348 & 2.1033 \\
\hline [13] & 1.8299 & 2.4993 & 1.7724 & 2.3044 & 1.3030 & 1.5526 & 1.4010 & 1.7199 & 1.7660 & 2.2984 & 1.7672 & 2.2909 \\
\hline [14] & 1.6007 & 2.0857 & 1.6130 & 2.0789 & 1.2401 & 1.4776 & 1.3232 & 1.6194 & 1.5938 & 2.0671 & 1.5858 & 2.0396 \\
\hline [15] & 1.7540 & 2.2729 & 1.6768 & 2.1690 & 1.2731 & 1.5170 & 1.3625 & 1.6695 & 1.6698 & 2.1834 & 1.6496 & 2.1262 \\
\hline [16] & 1.7848 & 2.3377 & 1.7207 & 2.2350 & 1.2532 & 1.4933 & 1.3429 & 1.6464 & 1.6982 & 2.2097 & 1.7251 & 2.2365 \\
\hline [17] & 1.7424 & 2.1501 & 1.6597 & 2.1438 & 1.2722 & 1.5159 & 1.3607 & 1.6669 & 1.6277 & 2.1042 & 1.6401 & 2.1126 \\
\hline
\end{tabular}


POTDAR \& SHIRKE

Table 3, continued.

\begin{tabular}{|c|c|c|c|c|c|c|c|c|c|c|c|c|}
\hline \multirow[b]{2}{*}{ Scheme } & \multicolumn{2}{|c|}{$\mathbf{C}_{1}$} & \multicolumn{2}{|c|}{$\mathbf{C}_{2}$} & \multicolumn{2}{|c|}{$\mathrm{C}_{3}$} & \multicolumn{2}{|c|}{$\mathrm{C}_{4}$} & \multicolumn{2}{|c|}{$\mathrm{C}_{5}$} & \multicolumn{2}{|c|}{$\mathrm{C}_{6}$} \\
\hline & $90 \%$ & $95 \%$ & $90 \%$ & $95 \%$ & $90 \%$ & $95 \%$ & $90 \%$ & $95 \%$ & $90 \%$ & $95 \%$ & $90 \%$ & $95 \%$ \\
\hline [18] & 1.7336 & 2.1373 & 1.6528 & 2.1345 & 1.2618 & 1.5035 & 1.3490 & 1.6523 & 1.6297 & 2.1138 & 1.6378 & 2.1099 \\
\hline [19] & 1.0242 & 1.2681 & 1.0099 & 1.2531 & 0.8758 & 1.0436 & 0.9047 & 1.0926 & 1.0153 & 1.2497 & 1.0011 & 2.2410 \\
\hline [20] & 1.0137 & 1.2284 & 0.9834 & 1.2145 & 0.8717 & 1.0387 & 0.8998 & 1.0864 & 0.9957 & 1.2302 & 0.9712 & 1.1978 \\
\hline [21] & 1.8246 & 2.3465 & 1.8066 & 2.3495 & 1.3169 & 1.5692 & 1.4194 & 1.7442 & 1.8067 & 2.3370 & 1.8018 & 2.3372 \\
\hline [22] & 1.6455 & 2.0421 & 1.6180 & 2.0857 & 1.2377 & 1.4748 & 1.3211 & 1.6170 & 1.6001 & 2.0816 & 1.5875 & 2.0391 \\
\hline [23] & 1.0462 & 1.2845 & 1.0328 & 1.2825 & 0.8884 & 1.0586 & 0.9189 & 1.1104 & 1.0393 & 1.2960 & 1.0311 & 1.2787 \\
\hline [24] & 1.0103 & 1.2819 & 0.9854 & 1.2171 & 0.8753 & 1.0430 & 0.9036 & 1.0911 & 0.9800 & 1.2079 & 0.9812 & 1.2099 \\
\hline [25] & 0.7842 & 0.9543 & 0.7775 & 0.9509 & 0.7016 & 0.8360 & 0.7165 & 0.8613 & 0.7766 & 0.9502 & 0.7754 & 0.9475 \\
\hline [26] & 0.7846 & 0.9490 & 0.7714 & 0.9407 & 0.7079 & 0.8435 & 0.7229 & 0.8691 & 0.7677 & 0.9354 & 0.7671 & 0.9342 \\
\hline [27] & 0.6895 & 0.8386 & 0.6832 & 0.8310 & 0.6328 & 0.7540 & 0.6436 & 0.7723 & 0.6820 & 0.8351 & 0.6820 & 0.8275 \\
\hline [28] & 0.6546 & 0.8045 & 0.6550 & 0.7944 & 0.6162 & 0.7343 & 0.6261 & 0.7510 & 0.6526 & 0.7914 & 0.6561 & 0.7941 \\
\hline [29] & 0.6009 & 0.7334 & 0.5902 & 0.7144 & 0.5567 & 0.6634 & 0.5640 & 0.6758 & 0.5945 & 0.7184 & 0.5879 & 0.7109 \\
\hline [30] & 0.5796 & 0.7047 & 0.5780 & 0.6982 & 0.5513 & 0.6569 & 0.5583 & 0.6688 & 0.5752 & 0.6973 & 0.5761 & 0.6951 \\
\hline [31] & 0.7042 & 0.8616 & 0.7249 & 0.8823 & 0.6713 & 0.7999 & 0.6842 & 0.8217 & 0.7312 & 0.8881 & 0.7259 & 0.8817 \\
\hline [32] & 0.6482 & 0.7763 & 0.6563 & 0.7960 & 0.6176 & 0.7359 & 0.6275 & 0.7526 & 0.6639 & 0.8022 & 0.6546 & 0.7929 \\
\hline [33] & 0.4067 & 0.4736 & 0.4067 & 0.4884 & 0.3951 & 0.4708 & 0.3977 & 0.4752 & 0.4043 & 0.4892 & 0.4047 & 0.4859 \\
\hline [34] & 0.3985 & 0.4815 & 0.3992 & 0.4789 & 0.3897 & 0.4644 & 0.3922 & 0.4686 & 0.4014 & 0.4818 & 0.3968 & 0.4754 \\
\hline
\end{tabular}

Table 4. Coverage to Length Ratio (CLR) of confidence intervals

\begin{tabular}{|c|c|c|c|c|c|c|c|c|c|c|}
\hline \multirow[b]{2}{*}{ Scheme } & \multicolumn{2}{|c|}{$C_{1}$} & \multicolumn{2}{|c|}{$\mathbf{C}_{3}$} & \multicolumn{2}{|c|}{$\mathbf{C}_{4}$} & \multicolumn{2}{|c|}{$\mathbf{C}_{5}$} & \multicolumn{2}{|c|}{$\mathbf{C}_{6}$} \\
\hline & $90 \%$ & $95 \%$ & $90 \%$ & $95 \%$ & $90 \%$ & $95 \%$ & $90 \%$ & $95 \%$ & $90 \%$ & $95 \%$ \\
\hline [1] & 0.3873 & 0.3026 & 0.5908 & 0.5180 & 0.5838 & 0.4988 & 0.4471 & 0.3582 & 0.4401 & 0.3497 \\
\hline [2] & 0.4119 & 0.3240 & 0.6046 & 0.5280 & 0.5892 & 0.5044 & 0.4705 & 0.3752 & 0.4667 & 0.3737 \\
\hline [3] & 0.4925 & 0.3854 & 0.6860 & 0.6002 & 0.6770 & 0.5840 & 0.5484 & 0.4460 & 0.5449 & 0.4433 \\
\hline [4] & 0.4977 & 0.4125 & 0.6845 & 0.6009 & 0.6754 & 0.5816 & 0.5675 & 0.4655 & 0.5759 & 0.4713 \\
\hline [5] & 0.3811 & 0.3007 & 0.5711 & 0.4992 & 0.5623 & 0.4766 & 0.4234 & 0.3305 & 0.4305 & 0.3423 \\
\hline [6] & 0.4130 & 0.3074 & 0.6033 & 0.5270 & 0.5871 & 0.5022 & 0.4689 & 0.3773 & 0.4680 & 0.3770 \\
\hline
\end{tabular}




\section{I. FOR HALF-LOGISTIC DISTRIBUTION UNDER TYPE-II CENSORING}

Table 4, continued.

\begin{tabular}{|c|c|c|c|c|c|c|c|c|c|c|}
\hline \multirow[b]{2}{*}{ Scheme } & \multicolumn{2}{|c|}{ C1 } & \multicolumn{2}{|c|}{ C3 } & \multicolumn{2}{|c|}{ C4 } & \multicolumn{2}{|c|}{ C5 } & \multicolumn{2}{|c|}{$\mathrm{C} 6$} \\
\hline & $90 \%$ & $95 \%$ & $90 \%$ & $95 \%$ & $90 \%$ & $95 \%$ & $90 \%$ & $95 \%$ & $90 \%$ & $95 \%$ \\
\hline [7] & 0.4489 & 0.3674 & 0.6541 & 0.5730 & 0.6442 & 0.5551 & 0.5256 & 0.4255 & 0.5287 & 0.4300 \\
\hline [8] & 0.4835 & 0.3807 & 0.6791 & 0.5943 & 0.6682 & 0.5739 & 0.5602 & 0.4618 & 0.5640 & 0.4627 \\
\hline [9] & 0.4977 & 0.3953 & 0.6725 & 0.5863 & 0.6591 & 0.5655 & 0.5609 & 0.4552 & 0.5493 & 0.4508 \\
\hline [10] & 0.4625 & 0.3832 & 0.6656 & 0.5788 & 0.6530 & 0.5649 & 0.5372 & 0.4356 & 0.5397 & 0.4425 \\
\hline [11] & 0.4791 & 0.3753 & 0.6684 & 0.5863 & 0.6641 & 0.5700 & 0.5343 & 0.4305 & 0.5510 & 0.4478 \\
\hline [12] & 0.4985 & 0.4036 & 0.6737 & 0.5916 & 0.6672 & 0.5718 & 0.5505 & 0.4488 & 0.5465 & 0.4510 \\
\hline [13] & 0.4546 & 0.3451 & 0.6460 & 0.5636 & 0.6311 & 0.5435 & 0.5097 & 0.4135 & 0.5073 & 0.4156 \\
\hline [14] & 0.5294 & 0.4222 & 0.6833 & 0.5979 & 0.6700 & 0.5769 & 0.5622 & 0.4583 & 0.5659 & 0.4639 \\
\hline [15] & 0.4771 & 0.3845 & 0.6589 & 0.5746 & 0.6423 & 0.5541 & 0.5374 & 0.4364 & 0.5461 & 0.4460 \\
\hline [16] & 0.4655 & 0.3693 & 0.6633 & 0.5802 & 0.6565 & 0.5624 & 0.5328 & 0.4314 & 0.5188 & 0.4246 \\
\hline [17] & 0.4839 & 0.4057 & 0.6675 & 0.5817 & 0.6519 & 0.5577 & 0.5532 & 0.4517 & 0.5487 & 0.4480 \\
\hline [18] & 0.4798 & 0.4066 & 0.6649 & 0.5824 & 0.6514 & 0.5604 & 0.5488 & 0.4489 & 0.5494 & 0.4503 \\
\hline [19] & 0.8389 & 0.7061 & 1.0037 & 0.8741 & 0.9840 & 0.8618 & 0.8825 & 0.7610 & 0.8941 & 0.7621 \\
\hline [20] & 0.8563 & 0.7382 & 0.9987 & 0.8759 & 0.9851 & 0.8614 & 0.9041 & 0.7745 & 0.9224 & 0.7863 \\
\hline [21] & 0.4492 & 0.3641 & 0.6287 & 0.5484 & 0.6174 & 0.5323 & 0.4976 & 0.4063 & 0.4982 & 0.4061 \\
\hline [22] & 0.5088 & 0.4270 & 0.6787 & 0.5907 & 0.6634 & 0.5754 & 0.5607 & 0.4584 & 0.5650 & 0.4661 \\
\hline [23] & 0.8258 & 0.7063 & 0.9721 & 0.8496 & 0.9640 & 0.8433 & 0.8637 & 0.7323 & 0.8709 & 0.7393 \\
\hline [24] & 0.8685 & 0.7121 & 1.0031 & 0.8756 & 0.9920 & 0.8646 & 0.9085 & 0.7836 & 0.9183 & 0.7862 \\
\hline [25] & 1.1112 & 0.9599 & 1.2500 & 1.0955 & 1.2488 & 1.0951 & 1.1493 & 0.9943 & 1.1614 & 0.9990 \\
\hline [26] & 1.1244 & 0.9705 & 1.2499 & 1.0957 & 1.2444 & 1.0935 & 1.1733 & 1.0148 & 1.1651 & 1.0135 \\
\hline [27] & 1.2827 & 1.1026 & 1.3891 & 1.2218 & 1.3850 & 1.2156 & 1.3199 & 1.1378 & 1.3153 & 1.1447 \\
\hline [28] & 1.3523 & 1.1562 & 1.4411 & 1.2654 & 1.4298 & 1.2610 & 1.3920 & 1.2045 & 1.3639 & 1.1959 \\
\hline [29] & 1.4818 & 1.2648 & 1.6077 & 1.4109 & 1.5996 & 1.4049 & 1.5180 & 1.3177 & 1.5220 & 1.3368 \\
\hline [30] & 1.5349 & 1.3265 & 1.6176 & 1.4270 & 1.6088 & 1.4181 & 1.5722 & 1.3668 & 1.5584 & 1.3635 \\
\hline [31] & 1.2667 & 1.0822 & 1.3192 & 1.1561 & 1.3099 & 1.1513 & 1.2319 & 1.0727 & 1.2354 & 1.0759 \\
\hline [32] & 1.3675 & 1.1988 & 1.4372 & 1.2687 & 1.4298 & 1.2594 & 1.3651 & 1.1885 & 1.3684 & 1.1954 \\
\hline [33] & 2.1957 & 1.9793 & 2.2622 & 1.9983 & 2.2625 & 1.9895 & 2.2158 & 1.9311 & 2.2351 & 1.9614 \\
\hline [34] & 2.2394 & 1.9556 & 2.3120 & 2.0353 & 2.3014 & 2.0320 & 2.2291 & 1.9611 & 2.2857 & 2.0076 \\
\hline
\end{tabular}




\section{POTDAR \& SHIRKE}

We denote by $\mathrm{C}_{1}$ the CI proposed by Balakrishnan and Asgharzadeh (2005), by $\mathrm{C}_{2}$ the CI proposed Wang (2009), by $\mathrm{C}_{3}$ the CI based on the MLE obtained by the $\mathrm{EM}$ algorithm, by $\mathrm{C}_{4}$ the $\mathrm{CI}$ based on the log-transformed MLE, by $\mathrm{C}_{5}$ the CI based on pivotal quantity, and by $\mathrm{C}_{6}$ the GCI. Coverage probabilities of the CIs for various censoring schemes are displayed in Table 2. Coverage probabilities of $\mathrm{C}_{1}$ are also displayed in the same table. Coverage probabilities for $\mathrm{C}_{2}$ are not provided by Wang (2009). Lengths of CIs for the various censoring schemes are given in Table 3. For comparison, lengths of $\mathrm{C}_{1}$ and $\mathrm{C}_{2}$ are given in the same table.

For effective comparison of CIs, we compute coverage to length ratio (CLR). CLR for $\mathrm{C}_{1}, \mathrm{C}_{3}, \mathrm{C}_{4}, \mathrm{C}_{5}$, and $\mathrm{C}_{6}$ are given in Table 4 . It is clear that the CIs having a higher value of CLR are preferred.

\section{Conclusion}

Coverage probabilities of $\mathrm{C}_{3}, \mathrm{C}_{4}, \mathrm{C}_{5}$, and $\mathrm{C}_{6}$ are better than coverage probabilities of $\mathrm{C}_{1}$. Comparing coverage probabilities of all four CIs, $\mathrm{C}_{5}$ and $\mathrm{C}_{6}$ show the best performance. For small and large sample sizes $(n)$ and the smallest effective sample size $(m), \mathrm{C}_{5}$ and $\mathrm{C}_{6}$ show good coverage probability. For large sample sizes, $\mathrm{C}_{3}, \mathrm{C}_{4}, \mathrm{C}_{5}$, and $\mathrm{C}_{6}$ show good performance. As $n$ and $m$ increase, coverage probability of $\mathrm{C}_{3}$ and $\mathrm{C}_{4}$ increases rapidly as compared to $\mathrm{C}_{5}$ and $\mathrm{C}_{6}$. $\mathrm{C}_{6}$ has higher coverage probability for conventional censoring schemes than progressive censoring schemes, but $\mathrm{C}_{3}$ and $\mathrm{C}_{4}$ show higher coverage probability for progressive censoring schemes than conventional censoring schemes.

$\mathrm{C}_{3}$ has smaller length than the lengths of $\mathrm{C}_{1}$ and $\mathrm{C}_{2}$. The MLE by the EM algorithm provides the shortest length $\mathrm{CI}$ among all five CIs. For large sample sizes, the length of $\mathrm{C}_{6}$ approaches the length of $\mathrm{C}_{3}$. Lengths of all CIs decrease as $n$ and $m$ increase. Lengths of CIs based on progressive censoring schemes are smaller than lengths of $\mathrm{CIs}$ based on conventional censoring schemes. There is a minor difference among lengths of $\mathrm{C}_{3}, \mathrm{C}_{4}, \mathrm{C}_{5}$, and $\mathrm{C}_{6}$ for large sample sizes. According to the CLR, $\mathrm{C}_{3}$ is the best among the four CIs for small sample sizes. $\mathrm{C}_{4}, \mathrm{C}_{5}$, and $\mathrm{C}_{6}$ also show higher CLR than the CLR of $\mathrm{C}_{1}$. CLRs of CIs based on

progressive censoring schemes are better than CLRs of CIs based on conventional censoring. 


\section{Acknowledgements}

The first author wishes to thank the University Grants Commission, New Delhi, India for providing fellowship under the Faculty Improvement Programme to carry out this research.

\section{References}

Balakrishnan, N. (2007). Progressive censoring methodology: An appraisal (with discussion). Test, 16(2), 211-296.doi: 10.1007/s11749-007-0061-y

Balakrishnan, N., \& Aggarwala, R. (2000). Progressive censoring: Theory, methods, and applications. Boston, MA: Birkhauser.doi: 10.1007/978-1-4612$1334-5$

Balakrishnan, N., \& Asgharzadeh, A. (2005). Inference for the scaled halflogistic distribution based on progressively Type-II censored samples. Communications in Statistics - Theory and Methods, 34(1), 73-87.doi: 10.1081/sta-200045814

Balakrishnan, N., \& Chan, P.S. (1992). Estimation for the scaled half logistic distribution under Type-II censoring.Computational Staistics \& Data Analysis, 13(2), 123-141.doi: 10.1016/0167-9473(92)90001-v

Balakrishnan, N., Kannan, N., Lin, C.T., \& Ng, H. K. T. (2003). Point and interval estimation for Gaussian distribution, based on progressively Type-II censored samples.IEEE Transactions on Reliability, 52(1), 90-95. doi:

$10.1109 /$ tr.2002.805786

Balakrishnan, N., Kannan, N., Lin, C.T., \& Wu, S. J. S. (2004). Inference for the extreme value distribution under progressive Type-II Censoring.Journal of Statistical Computationand Simulation, 74(1), 25-45.doi: 10.1080/0094965031000105881

Balakrishnan, N., \&Puthenpura, S. (1986). Best linear unbiased estimation of location and scale parameters of the half logistic distribution.Journal of Statistical Computation and Simulation, 25(3-4), 193-204.doi:

10.1080/00949658608810932

Balakrishnan, N., \& Sandhu, R. A. (1995). A simple simulation algorithm for generating progressive Type-II censored samples.The American Statistician, 49(2), 229-230.doi: 10.2307/2684646 


\section{POTDAR \& SHIRKE}

Balakrishnan, N., \& Wong, K. H. T. (1991). Approximate MLEs for the location and scaled parameters of the half logistic distribution with Type-II right censoring.IEEE Transactions on Reliability, 40(2), 140-145. doi:

$10.1109 / 24.87114$

Cohen, A. C. (1963). Progressively censored samples in life testing.Technometrics, 5(3), 327-329.doi: 10.2307/1266337

Dempster, A. P., Laird, N. M., \& Rubin, D. B. (1977). Maximum likelihood from incomplete data via the EM algorithm.Journal of the Royal Statistical Society. Series B (Methodological), 39(1), 1-38. Available from: http://www.jstor.org/stable/2984875

Ghitany, M. E., Alqallaf, F., \& Balakrishnan, N. (2014). On the likelihood estimation of the parameters of Gompertz distribution based on complete and progressively Type-II censored samples. Journal of Statistical Computation and Simulation, 84(8), 1803-1812.doi: 10.1080/00949655.2013.766738

Gulati, S., \& Mi, J. (2006). Testing for scale families using total variation distance.Journal of Statistical Computation and Simulation, 76(9), 773-792.doi: 10.1080/10629360500282080

Jang, D. H., Park, J., \& Kim, C. (2011). Estimation of the scale parameter of the half-logistic distribution with multiply type II censored sample.Journal of the Korean Statistical Society, 40(3), 291-301.doi: 10.1016/j.jkss.2010.12.001

Kim, C., \& Han, K. (2010). Estimation of the scale parameter of the halflogistic distribution under progressively typeII censored sample.Statistical Papers, 51(2), 375-387.doi: 10.1007/s00362-009-0197-9

Lawless, J. F. (1982).Statistical models and methods for lifetime data.New York, NY: John Wiley and Sons.

Lin, C.-T., \& Balakrishnan, N. (2011). Asymptotic properties of maximum likelihood estimators based on progressively Type-II censoring.Metrika, 74(3), 349-360.doi: 10.1007/s00184-010-0306-8

Louis, T. A. (1982). Finding the observed information matrix using the EM algorithm.Journal of the Royal Statistical Society. Series B (Methodological), 44(2), 226-233. Available from: http://www.jstor.org/stable/2345828

Mann, N. R. (1969). Exact three-order-statistic confidence bounds on reliable life for a Weibull model with progressive censoring. Journal of the American Statistical Association, 64(325), 306-315.doi: 10.2307/2283740

Mann, N. R. (1971). Best linear invariant estimation for Weibull parameters under progressive censoring.Technometrics, 13(3), 521-533.doi: 10.2307/1267165 


\section{I. FOR HALF-LOGISTIC DISTRIBUTION UNDER TYPE-II CENSORING}

McLachlan, G. J., \& Krishnan, T. (1997).The EM algorithm and extensions.New York, NY: John Wiley and Sons.

Meeker, W. Q., \& Escobar, L. A. (1998).Statistical methods for reliability data.New York, NY: John Wiley and Sons.

$\mathrm{Ng}, \mathrm{H}$. K. T. (2005). Parameter estimation for a modified Weibull distribution for progressively Type-II censored samples.IEEE Transactions on Reliability, 54(3), 374-380.doi: 10.1109/tr.2005.853036

Ng, H. K. T., Kundu, D., \& Balakrishnan, N. (2006). Point and interval estimation for the two-parameter Birnbaum-Saunders distribution based on progressively Type-II censored data.Computational Statistics \& Data Analysis, 50(11), 3222-3242.doi: 10.1016/j.csda.2005.06.002

Potdar, K. G., \& Shirke, D. T. (2013). Reliability estimation for the distribution of a $k$-unit parallel system with Rayleigh distribution as the component life distribution.International Journal of Engineering Research and Technology, 2(8), 2362-2371.

Potdar, K. G., \& Shirke, D. T. (2014). Inference for the scale parameter of lifetime distribution of $k$-unit parallel system based on progressively censored data. Journal of Statistical Computation and Simulation, 84(1), 171-185.doi: 10.1080/00949655.2012.700314

Rastogi, M. K., \& Tripathi, Y. M. (2014). Parameter and reliability estimation for an exponentiated half-logistic distribution under progressive type II censoring.Journal of Statistical Computation and Simulation, 84(8), 1711-1727. doi: 10.1080/00949655.2012.762366

Sultan, K. S., Alsadat, N. H., \& Kundu, D. (2014). Bayesian and maximum likelihood estimations of the inverse Weibull parameters under progressive typeII censoring. Journal of Statistical Computation and Simulation, 84(10), 22482265.doi: 10.1080/00949655.2013.788652

Wang, B. (2009). Interval estimation for the scaled half logistic distribution under progressive Type-II censoring.Communicationsin Statistics - Theory and Methods, 38(3), 364-371.doi: 10.1080/03610920802213681

Weerahandi, S. (1993). Generalized confidence intervals. Journal of the American Statistical Association, 88(423), 899-905.doi: 10.2307/2290779 


\section{Appendix A. Illustrative Examples}

\section{Numeric Example}

Balakrishnan and Asgharzadeh (2005) gave simulated sample of size $n=50$ from the half-logistic distribution with scale parameter $\lambda=25$. This complete sample is

$1.7110,2.0024,2.3963,3.9034,4.6412,6.4002,6.7956,8.5646,8.6428,8.8354$, $9.3518,9.7358,10.5080,10.5095,11.8015,12.8005,16.3451,16.9938,17.2101$, $18.5384,20.3508,21.1838,22.1529,22.4062,22.4381,23.0369,25.8435$, $27.0574,27.1237,29.0360,30.6449,32.5713,33.6688,40.3890,45.4092$, 46.4756, 49.8833, 51.1798, 53.0397, 53.8135, 64.9315, 66.1807, 69.9004, 75.2674, 75.4427, 75.7291, 76.1571, 89.5827, 99.8525, 134.6488.

Balakrishnan and Asgharzadeh (2005) and Wang (2009) derived CIs for this complete sample and the censored sample. We also derive CIs by using the MLE obtained by the EM algorithm, and the CIs based on pivot and generalized pivot. In Table 5, we consider two cases suggested by Wang (2009). Also we use the censoring schemes and samples given by Wang (2009) and derive 90\% and $95 \%$ CIs and their lengths. For comparison, we display CIs and their lengths as stated by Wang (2009).

Table 5. Confidence interval and its length for illustrative example: $n=50, \lambda=25$

\begin{tabular}{|c|c|c|c|c|}
\hline \multirow[b]{2}{*}{ Scheme } & \multicolumn{2}{|c|}{$\mathbf{C}_{2}$} & \multicolumn{2}{|c|}{$\mathrm{C}_{3}$} \\
\hline & $90 \%$ & $95 \%$ & $90 \%$ & $95 \%$ \\
\hline Case 1 & $(24.49,42.97)$ & $(23.37,45.72)$ & $(22.76,40.26)$ & $(21.08,41.94)$ \\
\hline$\left(25^{\star} 1\right)$ & 18.48 & 22.35 & 17.50 & 20.86 \\
\hline Case 2 & $(20.93,34.82)$ & $(20.05,36.81)$ & $(19.95,33.28)$ & $(18.67,34.56)$ \\
\hline \multirow[t]{2}{*}{$\left(28^{*} 0,10,10\right)$} & 13.89 & 16.76 & 13.33 & 15.89 \\
\hline & \multicolumn{2}{|c|}{$C_{5}$} & \multicolumn{2}{|c|}{$\mathbf{C}_{6}$} \\
\hline Scheme & $90 \%$ & $95 \%$ & $90 \%$ & $95 \%$ \\
\hline Case 1 & $(24.52,42.94)$ & $(23.38,45.67)$ & $(24.05,42.82)$ & $(23.18,45.66)$ \\
\hline$\left(25^{\star} 1\right)$ & 18.42 & 22.29 & 18.77 & 22.48 \\
\hline Case 2 & $(21.21,35.21)$ & $(20.31,37.23)$ & $(21.42,34.93)$ & $(20.31,37.24)$ \\
\hline$\left(28^{*} 0,10,10\right)$ & 14.00 & 16.92 & 13.51 & 16.93 \\
\hline
\end{tabular}

Note: For Case 1, Sr. No. is 1 and $m=25$. For Case 2, Sr. No. is 2 and $m=30$. 


\section{I. FOR HALF-LOGISTIC DISTRIBUTION UNDER TYPE-II CENSORING}

Table 6. Confidence interval and its length for illustrative example: $n=50, \lambda=25$

\begin{tabular}{|c|c|c|c|c|}
\hline \multirow[b]{2}{*}{ Scheme } & \multicolumn{2}{|c|}{$C_{1}$} & \multicolumn{2}{|c|}{$C_{3}$} \\
\hline & $90 \%$ & $95 \%$ & $90 \%$ & $95 \%$ \\
\hline Case 1 & $(19.81,29.53)$ & $(18.90,30.45)$ & $(19.88,29.48)$ & $(18.96,30.40)$ \\
\hline$(50 * 0)$ & 9.72 & 11.55 & 9.6 & 11.44 \\
\hline Case 2 & $(20.78,32.12)$ & $(19.72,33.18)$ & $(18.88,29.21)$ & $(17.89,30.20)$ \\
\hline$\left(39^{\star} 0,10\right)$ & 11.34 & 13.46 & 10.33 & 12.31 \\
\hline Case 3 & $(18.66,31.16)$ & $(17.48,32.34)$ & $(15.92,26.62)$ & $(14.89,27.65)$ \\
\hline \multirow[t]{2}{*}{$\left(29^{*} 0,20\right)$} & 12.5 & 14.86 & 10.7 & 12.76 \\
\hline & \multicolumn{2}{|c|}{$C_{5}$} & \multicolumn{2}{|c|}{$\mathbf{C}_{6}$} \\
\hline Scheme & $90 \%$ & $95 \%$ & $90 \%$ & $95 \%$ \\
\hline Case 1 & $(20.59,30.37)$ & $(19.85,31.60)$ & $(20.55,30.26)$ & $(19.92,31.28)$ \\
\hline$(50 * 0)$ & 9.78 & 11.75 & 9.71 & 11.36 \\
\hline Case 2 & $(19.68,30.38)$ & $(18.94,31.81)$ & $(19.53,30.07)$ & $(18.95,31.47)$ \\
\hline$\left(39^{\star} 0,10\right)$ & 10.7 & 12.87 & 10.54 & 12.52 \\
\hline Case 3 & $(16.95,28.23)$ & $(16.23,29.80)$ & $(16.90,28.20)$ & $(16.06,29.92)$ \\
\hline$\left(29^{\star} 0,20\right)$ & 11.28 & 13.57 & 11.3 & 13.86 \\
\hline
\end{tabular}

Note: For Case 1, Sr. No. is 1 and $m=50$. For Case 2, Sr. No. is 2 and $m=40$. For Case 3, Sr. No. is 3 and $m=30$.

Balakrishnan and Asgharzadeh (2005) considered three cases, $(n=50$, $m=50),(n=50, m=40)$, and $(n=50, m=30)$. They used progressive and conventional Type-II censored samples but have not provided samples. To compare the proposed CIs with the CI proposed by Balakrishnan and Asgharzadeh (2005), we considered conventional censored and complete samples considered by Balakrishnan and Asgharzadeh (2005). We obtained 90\% and 95\% CIs for these schemes. In Table 6, 90\% and 95\% CIs and their lengths are displayed. Also, the CIs and their length proposed by Balakrishnan and Asgharzadeh (2005) are displayed.

Observe that in the illustrated example, $\mathrm{C}_{3}$ has shorter length than the lengths of $\mathrm{C}_{1}, \mathrm{C}_{2}$ and $\mathrm{C}_{5} . \mathrm{C}_{6}$ has shorter length than that of $\mathrm{C}_{1}$.

\section{Real Data Example}

Lawless (1982) presented real data which represented failure times for a specific type of electrical insulation that was subjected to a continuously increasing voltage stress.

$12.3,21.8,24.4,28.6,43.2,46.9,70.7,75.3,95.5,98.1,138.6,151.9$. 


\section{POTDAR \& SHIRKE}

Table 7. Confidence interval and its length for real data: $n=12, \lambda=50.50$ (BLUE)

\begin{tabular}{|c|c|c|c|c|}
\hline \multirow[b]{2}{*}{ Scheme } & \multicolumn{2}{|c|}{$\mathrm{C}_{3}$} & \multicolumn{2}{|c|}{$\mathrm{C}_{4}$} \\
\hline & $90 \%$ & $95 \%$ & $90 \%$ & $95 \%$ \\
\hline Case 1 & $(28.59,66.24)$ & $(24.98,69.85)$ & $(31.88,70.53)$ & $(29.54,76.10)$ \\
\hline$\left(12^{*} 0\right)$ & 37.65 & 44.87 & 38.65 & 46.56 \\
\hline Case 2 & $(25.55,73.70)$ & $(20.94,78.31)$ & $(30.55,80.61)$ & $(27.84,88.46)$ \\
\hline$\left(7^{\star} 0,4\right)$ & 48.15 & 57.37 & 50.06 & 60.62 \\
\hline Case 3 & $(23.35,68.29)$ & $(19.05,72.59)$ & $(28.06,74.82)$ & $(25.54,82.19)$ \\
\hline \multirow[t]{2}{*}{$\left(4,7^{*} 0\right)$} & 44.94 & 53.54 & 46.74 & 56.65 \\
\hline & \multicolumn{2}{|c|}{$\mathrm{C}_{5}$} & \multicolumn{2}{|c|}{$\mathrm{C}_{6}$} \\
\hline Scheme & $90 \%$ & $95 \%$ & $90 \%$ & $95 \%$ \\
\hline Case 1 & $(33.37,75.18)$ & $(31.19,82.30)$ & $(33.65,73.96)$ & $(31.88,83.36)$ \\
\hline$\left(12^{\star} 0\right)$ & 41.81 & 51.11 & 40.31 & 51.48 \\
\hline Case 2 & $(33.13,90.13)$ & $(30.73,101.89)$ & $(32.60,86.50)$ & $(30.13,94.26)$ \\
\hline$\left(7^{\star} 0,4\right)$ & 57 & 71.16 & 53.9 & 64.13 \\
\hline Case 3 & $(30.14,82.01)$ & $(27.78,92.25)$ & $(30.55,83.15)$ & $(27.58,92.42)$ \\
\hline$\left(4,7^{\star} 0\right)$ & 51.87 & 64.47 & 52.6 & 64.84 \\
\hline
\end{tabular}

Note: For Case 1, Sr. No. is 1 and $m=12$. For Case 2, Sr. No. is 2 and $m=8$. For Case 3, Sr. No. is 3 and $m=8$.

The half-logistic distribution fits the data extremely well (Balakrishnan \& Chan, 1992). This dataset was used with two censoring schemes, $(7 * 0,4)$ and $\left(4,7^{*} 0\right)$, and complete data, and the CI is constructed based on the MLE, logMLE, pivot, and generalized pivot. These $90 \%$ and $95 \%$ CIs and their lengths are presented in Table 7. Observe that, for real data, $\mathrm{C}_{3}$ has shorter length than $\mathrm{C}_{4}, \mathrm{C}_{5}$ and $\mathrm{C}_{6}$.

The EM algorithm approach works well for small sample size $n$ and the smallest effective sample size $m$. Overall, the proposed CIs perform better than the CIs proposed by Balakrishnan and Asgharzadeh (2005) and Wang (2009). The proposed CIs are superior to the other two CIs with regard to the length and the coverage probability. 